\title{
Effect of small wearable device antenna location on its impedance, bandwidth potential and radiation efficiency
}

\author{
J. Chen, M. Berg, H. Y. Amin, A. Pärssinen \\ Centre for Wireless Communications - University of Oulu, Finland, jiangcheng.chen@oulu.fi
}

Keywords: Internet of Things (IoT), user interaction, Wearable antennas, inverted $\mathrm{F}$ antennas (IFAs)

\begin{abstract}
In this paper, a simulation study of the effect of antenna locations on antenna matching, bandwidth potential and radiation efficiency performance operating in 3GPP Long Term Evolution (LTE) Band 3 (1710-1880 MHz), Band 20 UL (832-862 MHz) and 2.4 GHz WLAN band (2.4-2.5 GHz), in free space and with phantom, is presented. An inverted $F$ antenna (IFA) is utilized in this study and mounted on a small cellular wrist device. The results indicate that for each band, with efficient excitation of its fundamental mode, an antenna whose electrical field maxima located at short edge of the chassis always has higher bandwidth potential and its radiation efficiency will have less reduction when introducing phantom. Moreover, changes in matching characteristic of different antenna positions vary from each band, indicating the optimal antenna placement to obtain robust matching feature. Therefore, by comparing the variations on antenna behaviors, it provides useful insights into how to select the optimal antenna locations on the device with reduced a user effect for a specific operation band.
\end{abstract}

\section{Introduction}

In these days, the evolution of LTE for LTE-M (enhanced Machine Type Communication) and NB-IoT (Narrow BandInternet of Things) enables Cellular IoT for low cost, low power and wide area deployments [1], [2]. Therefore, it can be seen that numerous services are envisioned for cellular IoT and the market of smart mobile and wearable devices, e.g. smart watch, are rapidly ever-increasing. Since smart wearable devices need the access to wireless data, antenna becomes an indispensable component.

However, the antenna performance in such a mobile terminal devices suffers from the user interaction such as mismatch and absorption [3]. A wide range of studies with respect to the effects of user's proximity on mobile terminal antenna performance have been carried out [3], [4], [5], [6][7][8]. In these studies, performance changes of antennas are investigated by introducing lossy dielectric materials to represent the head and hand with different grips located at close vicinity, which, on the other hand, provide insufficient knowledge to deal with novel wearable applications. Due to the different configurations of antenna and phantom placements in comparison with mobile phone terminals above, and due to much smaller device dimensions, the previous studies are not directly applicable wearable devices. The low band cellular system frequencies are well below the resonance frequency of the device ground plane, which is the main source of radiation at these frequencies. Thus, there is a need to redefine the study of user interaction on the wearable device antenna performance.

Although some antenna designs with wideband behaviour based on wristband are proposed for LTE low band [9], the low radiation efficiency (below $-10 \mathrm{~dB}$ ) caused by the absorption makes it less likely to apply to cellular IoT applications. Given that, in this paper, IFAs are adopted as LTE-M low band and mid band as well as $2.4 \mathrm{GHz} \mathrm{Wi}-\mathrm{Fi}$ (denoted as high band in this paper) antennas inside the watch casing, providing higher efficiency performance at the expense of bandwidth. In particular, low band IFA can be designed as a tunable antenna [10] to cover the whole spectrum over $699 \mathrm{MHz}$ to $862 \mathrm{MHz}$. In this work, LTE Band 20 up link (UL), is chosen to demonstrate the user interaction on low band antenna.

This paper is to explore effects of various antenna locations and phantom on antenna performance over three different operating bands: Band 3 and Band 20 UL selected for LTE-M application and $2.4 \mathrm{GHz} \mathrm{Wi}-\mathrm{Fi}$ band. These can provide antenna designers with useful knowledge to select the optimal antenna placement for a given operation band. The antenna configurations for cellular IoT and Wi-Fi as well as different simulation cases used in this investigation are proposed. The simulated results for the variations of antenna performance, with respect to matching level, bandwidth potential and radiation efficiency, in various cases are presented.

\section{Description of the experiment}

\section{A. antenna structure and simulation models}

The simulations are carried out using time domain solver of CST Microwave Studio. A simple smartwatch model shown in Fig. 1(a) and (b), including the casing, battery, display, chassis and antenna, is established to evaluate the performance of various antenna placements in free space and with phantom. The forearm-like phantom is placed underneath the casing to mimic the wrist interacted with the small terminal. Due to the limited space inside the casing, antennas in our study are implemented on the top edge of the casing, making them conformal to the casing. The lengths of antenna for Band 3, Band $20 \mathrm{UL}$ and WLAN band are 82 
$\mathrm{mm}, 32.5 \mathrm{~mm}$ and $23.5 \mathrm{~mm}$ respectively. In order to tune the antenna resonant frequency of the operation band, each antenna length is slightly modified.

The property of each material in these simulations is set as following: plastic casing is set with permittivity $\varepsilon_{\mathrm{r}}$ of 3 and loss tangent of 0.015 ; battery, display, chassis and antenna element are set as copper with conductivity $\sigma=5.96 \times 10^{7}$ $\mathrm{S} / \mathrm{m}$; phantoms are employed with $\varepsilon_{\mathrm{r}}=30.3$ and $\sigma=0.59 \mathrm{~S} / \mathrm{m}$ at $835 \mathrm{MHz}, \varepsilon_{\mathrm{r}}=27$ and $\sigma=0.99 \mathrm{~S} / \mathrm{m}$ at $1800 \mathrm{MHz}$ and $\varepsilon_{\mathrm{r}}=$ 25.7 and $\sigma=1.32 \mathrm{~S} / \mathrm{m}$ at $2450 \mathrm{MHz}[11]$.

The dimensions of the mobile terminal used in this simulation are given as follows. The volume of display, battery and chassis are $30.4 \times 26.11 \times 0.1 \mathrm{~mm}^{3}, 27.5 \times 25.9 \times 3.4 \mathrm{~mm}^{3}$ and $45 \times 30 \times 1.524 \mathrm{~mm}^{3}$ respectively. The casing is modelled hollow box without cover and its dimension is provided as $52 \times 37 \times 9.5 \mathrm{~mm}^{3}$ and the wall thickness is 2 $\mathrm{mm}$. Furthermore, the chassis is placed $2.5 \mathrm{~mm}$ above the phantom.

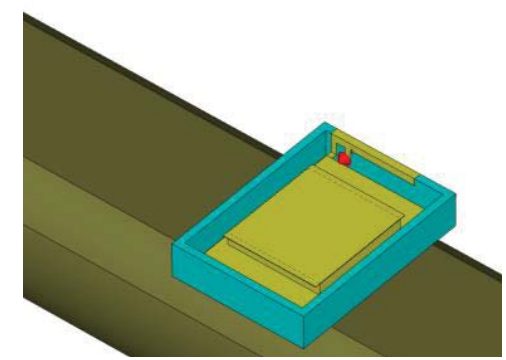

(a)

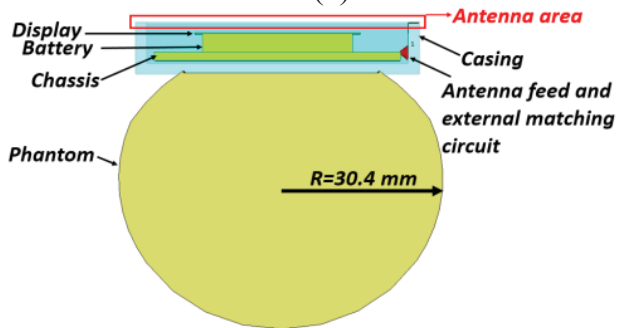

(b)

Fig. 1. (a) Overview of the wrist device antenna structure; (b) Cross-sectional view of the antenna configuration.

\section{B. Simulation Set-up}

In this paper, a substantial set of simulation series are performed to attest how the antenna performance, namely matching, bandwidth and radiation efficiency, are affected by various antenna positions and interacted with the phantom, over Band 3, Band $20 \mathrm{UL}$ and $2.4 \mathrm{GHz}$ WLAN band. Specifically, for each case, only single antenna is implemented on the casing. Moreover, for each operation band, there are four antenna locations simulated both in free space and with phantom respectively see Fig. 2(a), (b) and (c). In general, the IFAs are fed at corners and middles along short edge and long edge of the chassis so as to involve possible antenna positions in common mobile terminal antenna design.

On the other hand, the matching performance evaluation can be divided into frequency shift $\left(\Delta \mathrm{f}_{\mathrm{r}}\right)$ and impedance behaviour. The frequency detuning $\left(\Delta \mathrm{f}_{\mathrm{r}}\right)$ denotes the frequency difference between the resonance of an antenna matched critically to $50 \mathrm{Ohm}$ in free space and that of the antenna with phantom using the same matching circuit as in free space, indicating how large the antenna is detuned by the user. In our study, the matching circuits are added externally to the port and calculated by Optenni Lab. Moreover, to compare the bandwidth performance of different antennas, bandwidth potential is used to demonstrate the obtainable bandwidth the antenna structure can achieve by a twocomponent matching circuit. Optenni Lab is used to calculate the bandwidth potential [12]. The matching criteria were $\mathrm{S}_{11}$ $<-6 \mathrm{~dB}$ and $<-10 \mathrm{~dB}$ for LTE and Wi-Fi, respectively.
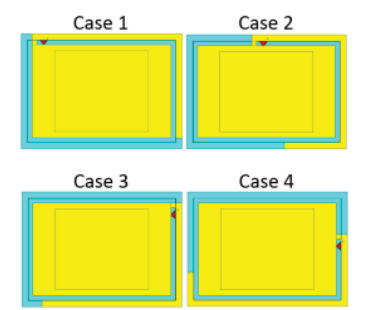

(a)

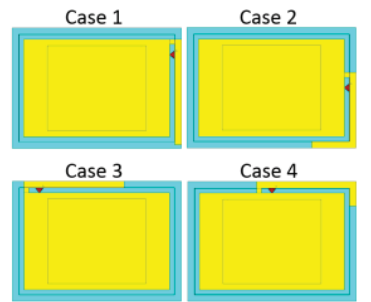

(b)

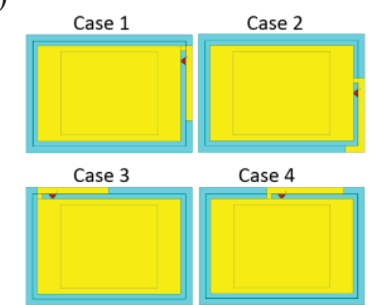

(c)
Fig. 2. Antenna placements for (a) Band 20 UL; (b) Band 3; (c) $2.4 \mathrm{GHz}$ WLAN Band.

\section{Simulation results}

\section{A. User influence on matching}

Fig. 3(a), (b) and (c) show the matching level variations with the presence of phantom for various cases operating in three bands and the corresponding detuning levels are summarized in Table 1. It is indicated from the Fig. 3(a) and Table 1 that the low band antenna is susceptible to the introduction of phantom: a large frequency detuning happens in all the investigated cases. Meanwhile, around center frequency, large impedance variations occur for all cases when introducing phantom. The impedances become less resistive and much more inductive for Case 1 and 2, whereas a reverse trend observed in Case 3 and 4.

In the Band 3, the frequency deviation of Case 1, 2 and 3 is small while slight shift occurs in Case 4, shown in Fig. 3(b) and Table 1. As observed, antennas in all cases for Band 3 are robust in frequency shift performance that the introduction of phantom results in slight impedance changes in Case 3 (less resistive and more inductive) as well as in Case 4 (more resistive and more capacitive), while the impedance behaviour in Case 3 and 4 remains almost stable.

It can be seen from Fig. 3(c), Case 1 and 2, that antennas placed at short edge have strong interaction with the wrist phantom such that the detuning level is larger than that of the remaining cases. Additionally, despite of similar antenna placements as in mid band, effects on impedance for four 
cases differ from those in $2.4 \mathrm{GHz}$. It is evident from the Fig. 3(c) that Case 1, 3 and 4 have less interaction with the phantom so that impedances keep virtually the same whilst impedance for Case 2 become less resistive.

Although the impedance changes vary from each band based on different antenna placements, it can be derived that the larger the mismatch level caused by the phantom, the more impedance variation happens. By selecting appropriate antenna position, the effect of the phantom on antenna performance can be reduced.

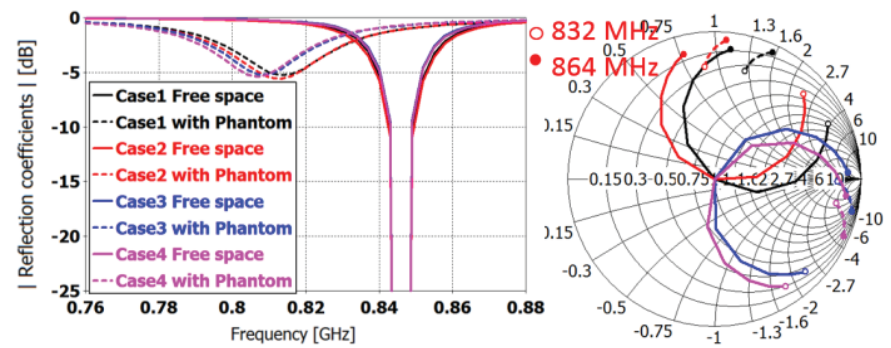

(a)

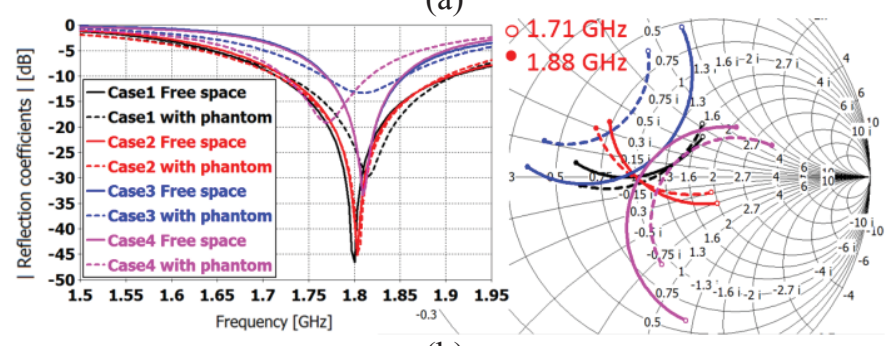

(b)

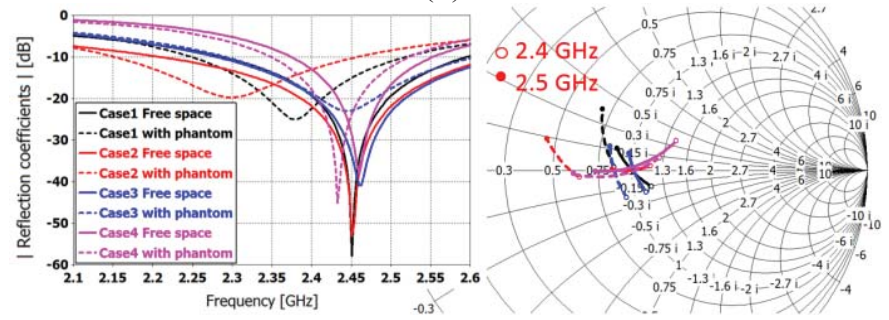

(c)

Fig. 3. Effect of the user proximity on resonant frequencies in (a) Band $20 \mathrm{UL}$, (b) Band 3 and (c) $2.4 \mathrm{GHz}$ WLAN Band

\section{B. User influence on bandwidth potential}

It is indicated in [13] that the optimal feed position using an IFA to couple into the first characteristic mode of a chassis is the short edge, especially at the corner, where stronger electrical field appears, and hence wider bandwidth performance is obtained by efficiently exciting the chassis mode [14].

In the low band, the chassis is much less than half wavelength of the operation band, $180 \mathrm{~mm}$, and hence it is too small to support the fundamental mode so that the radiation relies less on the chassis and the current distributions are more localized at the vicinity of antenna elements in all cases shown in Fig. 4(a). For this reason, the bandwidths become quite narrow as implied in Fig. 5(a). The bandwidth potential levels maintain nearly the same in all cases and they are increased by three times with the introduction phantom in contrast to those in free space. The decrease of Q-factor value, caused by resistive losses of the lossy dielectric material, accounts for the enhancement of bandwidth [15].

\begin{tabular}{|c|c|c|}
\hline \multicolumn{2}{|c|}{ Case \# } & $\left|\Delta f_{r}\right|[\%]$ \\
\hline \multirow{3}{*}{ Band 20 } & 1 & 3.9 \\
\cline { 2 - 3 } UL & 2 & 3.9 \\
\cline { 2 - 3 } & 3 & 4.5 \\
\cline { 2 - 3 } & 4 & 4.6 \\
\cline { 2 - 3 } Band 3 & 1 & 0.8 \\
\cline { 2 - 3 } & 2 & 0.1 \\
\cline { 2 - 3 } & 3 & 0.01 \\
\hline \multirow{3}{*}{$2.4 \mathrm{GHz}$} & 4 & 2.1 \\
\cline { 2 - 3 } WLAN & 1 & 2.9 \\
\cline { 2 - 3 } Band & 2 & 6.1 \\
\cline { 2 - 3 } & 3 & 0.7 \\
\hline
\end{tabular}

Table 1: Frequency detuning

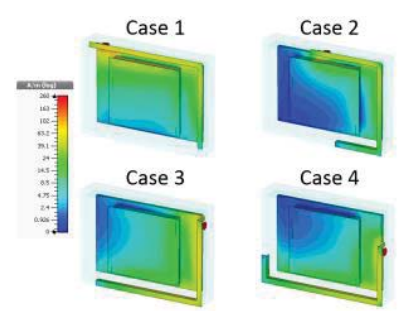

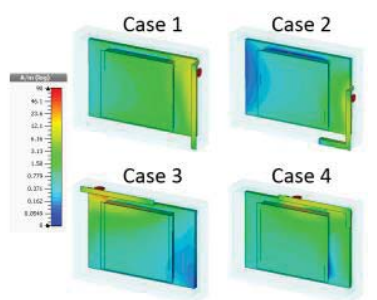

(b) (a)

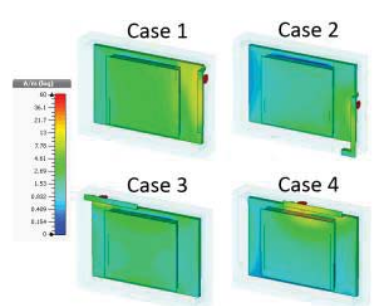

(c)
Fig. 4. Current distributions at (a) $846 \mathrm{MHz}$, (b) $1.8 \mathrm{GHz}$ and (c) $2.45 \mathrm{GHz}$ in free space

As can be seen from Fig. 5(b), both in fee space and with wrist phantom, Case 1 and 2 have higher bandwidth potential over the operation band wherein Case 2 has the highest bandwidth potential, while Case 3 and 4 perform worse. This can be explained by theory of characteristic mode (TCM) that in either Case 1 or 2 , due to the successful excitation of first chassis mode by locating the IFA nearby the short edge, the chassis acts as effective radiator and its surface current is distributed over the whole chassis which is similar to the fundamental chassis mode. In turn, the antennas placed in such position have larger bandwidth potential [13]. The current distribution in Case 3 and 4, compared with cases abovementioned, is more localized to antenna vicinity, leading to lower bandwidth level. With the maxima of electrical field distribution of the IFA locates at the corner, Case 2 has the highest bandwidth performance. Furthermore, levels in free space remain approximately the same in Case 1 and 2 , and are deteriorated substantially by the phantom while the bandwidth level is slightly improved by the phantom in Case 3 and 4 . It can be inferred that the variation of 
bandwidth potential for a certain antenna position depends on the variations on impedance.

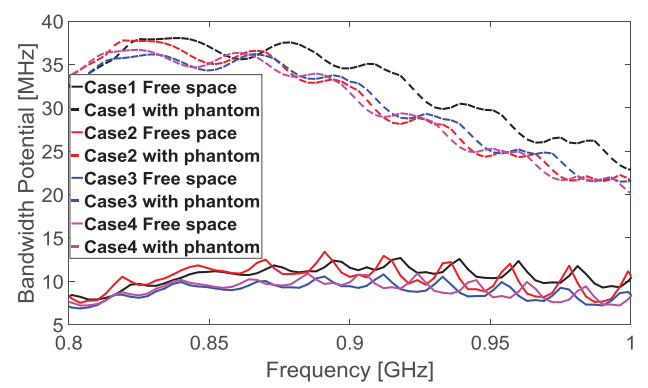

(a)

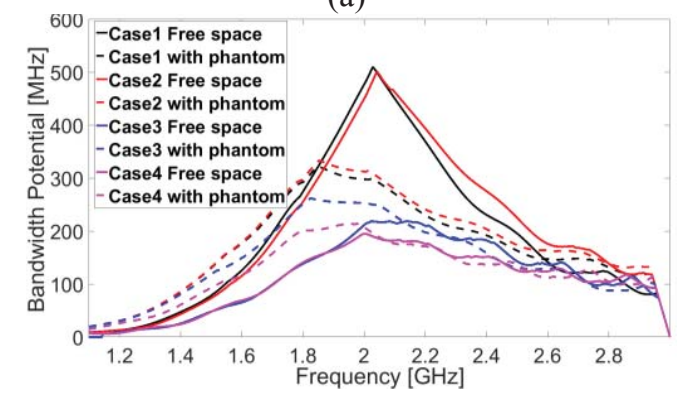

(b)

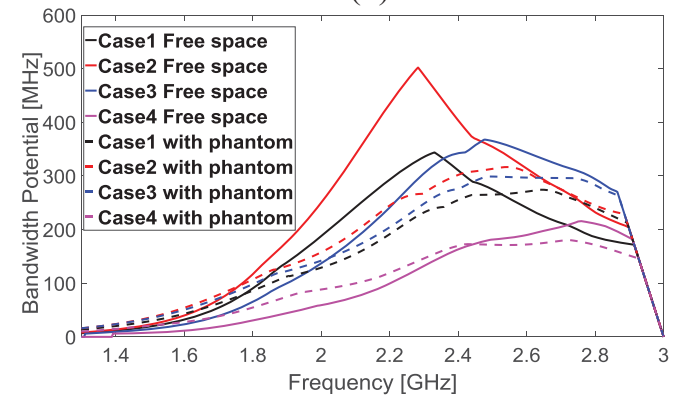

(c)

Fig. 5. Effect of the user proximity on bandwidth potential performance in (a) Band $20 \mathrm{UL}$, (b) Band 3 and (c) $2.4 \mathrm{GHz}$ WLAN Band

For $2.4 \mathrm{GHz}$ band, the current distribution of each case is provided in Fig. 4(c). A similar observation is obtained that Case 1 and 2, of which antennas are placed at short edge, has the optimal bandwidth property, see Fig. 5(c). The reason for the decent performance is the same as cases in $1.8 \mathrm{GHz}$ band with similar current distributions. In addition, the current distribution of Case 3 differs from that of Case 3 at $1.8 \mathrm{GHz}$. Although both cases have same antenna placement, Case 3 also appear good bandwidth potential as Case 1 and 2, resulting from the more intensive electrical field distribution around the corner at $2.45 \mathrm{GHz}$. Similarly, the degradation level can also be derived from impedance variations showed in Fig. 3(c): the larger the variation on impedance, the more the degradation is.

Careful selection of feed position and efficient activation of chassis mode are of great significance to determine the bandwidth performance. It is also interesting to note that the maxima of each bandwidth potential curve is not aligned with the resonant frequency. The maxima of the curve is dependent on the resonant behavior of the terminal chassis, namely the effective electrical dimension of the radiator (ground plane and antenna element). For example, with similar physical dimension of chassis and antenna element, the frequencies of maxima for Case 2 and Case 4 at $2.45 \mathrm{GHz}$ Band are $2.25 \mathrm{GHz}$ and $2.75 \mathrm{GHz}$ respectively, corresponding to different effective electrical lengths. Additionally, the phantom has effect on the effective electrical length of the chassis as observed in Fig. 5(a), (b) and (c) that maxima of bandwidth against frequency shifts from case to case. Given that, it is possible to shift the maxima of bandwidth potential to a desired operating frequency by slightly modify the radiator geometry, thereby maximizing the antenna bandwidth potential at the specific frequency. Also, this can also be used to compensate for the effect of phantom on the resonance by predicting the change of effective electrical dimension.

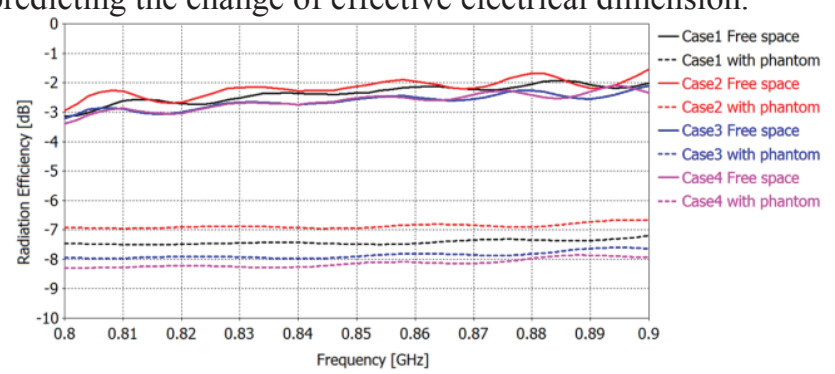

(a)

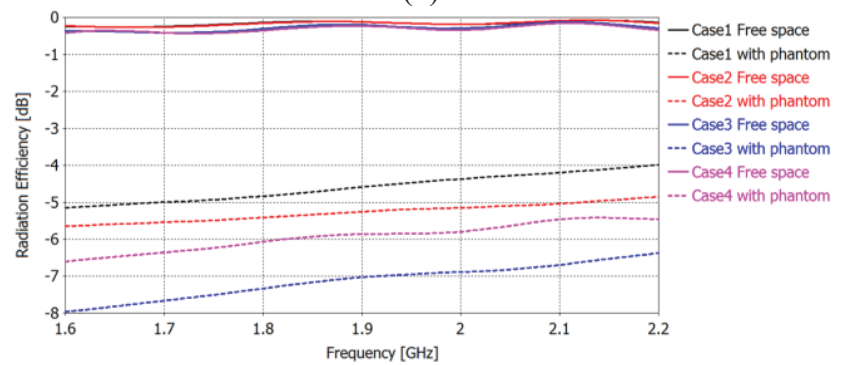

(b)

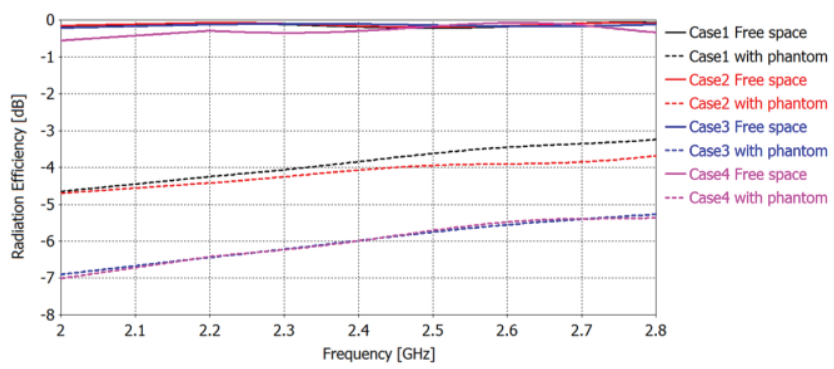

(c)

Fig. 6. Effect of the user proximity on Radiation efficiencies in (a) Band 20 UL, (b) Band 3 and (c) $2.4 \mathrm{GHz}$ WLAN Band

\section{User influence on radiation efficiency}

The phantom in this study mainly interfere with the electrical fields of antennas and radiation efficiency is greatly affected by human tissue. Therefore, it can be anticipated that the larger area the electrical field interacted with the phantom, the greater the reduction is in the radiation efficiency.

It can be seen clearly from Fig. 6 (a), (b) and (c) that in free space antennas in all cases have similar efficiency level over their operation bands and the radiation efficiencies for all cases are degraded to various extents when introducing phantom. In Fig. 6(a), the lowest reduction occurs in Case 2, 
whereas the greatest absorption happens in Case 4, which agrees with the prediction above. Interactions of the antenna Electrical field with the phantom showed in Fig.7. Same observations are obtained in Band 3 and $2.45 \mathrm{GHz}$ Band (see Fig. 6(b) and (c)). For those cases operating in characteristic mode, the strongest electrical fields are distributed along the short edge, causing less disturbance in electrical fields when loading a lossy dielectric material. The radiation efficiencies, as a result, are degraded less than their counterparts in remaining cases. Overall, antennas placed at the short edge have better radiation performance.

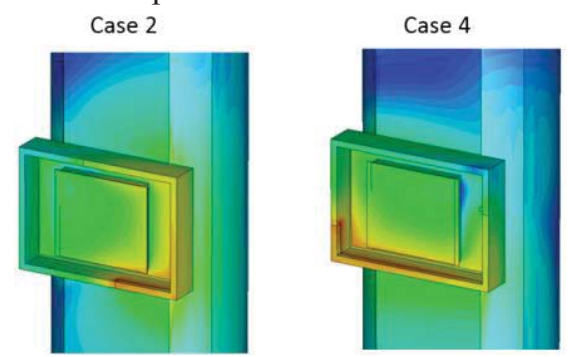

Fig. 7. Electrical field distributions with phantom

\section{Conclusions}

This paper investigates various IFA locations with the user interaction on the antenna performance in terms of frequency shift, impedance behavior, bandwidth potential and radiation efficiency, operating in LTE-M low- and mid-band as well as 2.4 GHz Wi-Fi band. The results show that the antenna location is a determinant of the antenna performance aforementioned as well as how the body tissue material disturbs the near field of different antenna placements with respect to the performance. In particular, with efficient excitation of its fundamental mode, an antenna whose electrical field maxima located at short edge of the chassis always has higher bandwidth potential and its radiation efficiency will have less reduction when introducing phantom. In addition, it is observed that while the bandwidth potential performance of mid- and high-band antennas are deteriorated, phantom can exert a positive influence on low band antenna. On the other hand, changes in matching characteristic of different antenna positions vary from each band. Therefore, by predicting the detuning and impedance behavior under the user body impact, the optimal antenna position for robust matching feature at a specific band can be derived and hence the adverse effects of loading a lossy dielectric material can be minimized.

In this way, depending on demands of the application, the study provides useful insights into how the antenna performance can be traded off with different antenna locations and with user at vicinity.

\section{References}

[1] R. Ratasuk, N. Mangalvedhe, A. Ghosh and B. Vejlgaard, "Narrowband LTE-M System for M2M Communication," 2014 IEEE 80th Vehicular Technology Conference (VTC2014-Fall), Vancouver, BC, 2014, pp.

[2] A. Díaz-Zayas, C. A. García-Pérez, Á. M. Recio-Pérez and P. Merino, "3GPP Standards to Deliver LTE
Connectivity for IoT," 2016 IEEE First International Conference on Internet-of-Things Design and Implementation (IoTDI), Berlin, 2016, pp. 283-288.

[3] M. Berg, M. Sonkki and E. Salonen, "Experimental study of hand and head effects to mobile phone antenna radiation properties," 2009 3rd European Conference on Antennas and Propagation, Berlin, 2009, pp. 437-440.

[4] K. R. Boyle, Y. Yuan and L. P. Ligthart, "Analysis of Mobile Phone Antenna Impedance Variations With User Proximity," in IEEE Transactions on Antennas and Propagation, vol. 55, no. 2, pp. 364-372, Feb. 2007.

[5] S. C. Del Barrio, M. Pelosi, O. Franek and G. F. Pedersen, "The Effect of the User's Body on High-Q and Low-Q Planar Inverted F Antennas for LTE Frequencies," 2012 IEEE 75th Vehicular Technology Conference (VTC Spring), Yokohama, 2012, pp. 1-4.

[6] J. Holopainen et al., "Equivalent circuit model-based approach on the user body effect of a mobile terminal antenna," 2010 Loughborough Antennas \& Propagation Conference, Loughborough, 2010, pp. 217-220.

[7] T. Huang and K. R. Boyle, "User Interaction Studies on Handset Antennas," The Second European Conference on Antennas and Propagation, EuCAP 2007, Edinburgh, 2007, pp.1-6.

[8] J. Ilvonen, O. Kivekas, J. Holopainen, R. Valkonen, K. Rasilainen and P. Vainikainen, "Mobile Terminal Antenna Performance With the User's Hand: Effect of Antenna Dimensioning and Location," in IEEE Antennas and Wireless Propagation Letters, vol. 10, no. , pp. 772$775,2011$.

[9] K. Zhao, Z. Ying and S. He, "Antenna designs of smart watch for cellular communications by using metal belt," 2015 9th European Conference on Antennas and Propagation (EuCAP), Lisbon, 2015, pp. 1-5.

[10] S. Caporal Del Barrio, M. Pelosi, O. Franek and G. F. Pedersen, "Tuning Range Optimization of a Planar Inverted F Antenna for the LTE Low Frequency Bands," 2011 IEEE Vehicular Technology Conference (VTC Fall), San Francisco, CA, 2011, pp. 1-5.

[11] Test plan for wireless device over-the-air performance rev. 3.6.1, CTIA Certification Standard, Nov. 2016.

[12] J. Rahola, "Bandwidth potential and electromagnetic isolation: Tools for analysing the impedance behaviour of antenna systems," 2009 3rd European Conference on Antennas and Propagation, Berlin, 2009, pp. 944-948.

[13] H. Li, Y. Tan, B. K. Lau, Z. Ying and S. He, "Characteristic Mode Based Tradeoff Analysis of Antenna-Chassis Interactions for Multiple Antenna Terminals," in IEEE Transactions on Antennas and Propagation, vol. 60, no. 2, pp. 490-502, Feb. 2012.

[14]R. Martens, E. Safin and D. Manteuffel, "Inductive and capacitive excitation of the characteristic modes of small terminals," 2011 Loughborough Antennas \& Propagation Conference, Loughborough, 2011, pp.1-4.

[15]A. D. Yaghjian and S. R. Best, "Impedance, bandwidth, and Q of antennas," in IEEE Transactions on Antennas and Propagation, vol. 53, no. 4, pp. 1298-1324, April 2005. 